\title{
THEORY OF SPACECRAFT DOPPLER TRACKING
}

\author{
MASSIMO TINTO \\ Jet Propulsion Laboratory \\ 4800 Oak Grove Drive, Pasadena, CA, U.S.A. \\ E-mail: Massimo.Tinto@jpl.nasa.gov
}

\begin{abstract}
We present a review of the spacecraft Doppler tracking technique used in broad band searches for gravitational waves in the millihertz frequency band. After deriving the transfer functions of a gravitational wave pulse and of the noise sources entering into the Doppler observable, we summarize the upper limits for the amplitudes of gravitational wave bursts, continuous, and of a stochastic background estimated by Doppler tracking experiments.
\end{abstract}

1. Introduction. Non-resonant detectors of gravitational radiation (with frequency content $0<f<f_{0}$ ) are essentially interferometers with one or more arms, in which a coherent train of electromagnetic waves (of nominal frequency $\nu_{0} \gg f_{0}$ ) is folded into several beams, and at points where these intersect relative fluctuations of frequency or phase are monitored (homodyne detection). Frequency fluctuations in a narrow band can alternatively be described as fluctuating sideband amplitudes, and interference of two or more beams, produced and monitored by a nonlinear device (such as a photo detector when light from a laser is used), exhibits these side bands as a low frequency signal again with frequency content $0<f<f_{0}$. The observed low frequency signal is due to frequency variations of the source of the beams about $\nu_{0}$, to relative motions of the source and the mirrors that do the folding (or the Earth and amplifying transponders), to temporal variations of the index of refraction along the beams, and, according to general relativity, to any time-variable gravitational fields present, such as the transverse traceless metric curvature of a passing plane gravitational wave train. To observe these gravitational fields in this way, it is thus necessary to control, or monitor, the other sources of relative frequency fluctuations, and, in the data analysis, to optimally use algorithms based on the different characteristic interferometer responses to gravitational waves (the signal) and to the other sources (the noise). Several feasibility studies [1-4] have shown that

1991 Mathematics Subject Classification: Primary 46C20; Secondary 32G81.

This work was performed at the Jet Propulsion Laboratory, California Institute of Technology, under a contract with the National Aeronautics and Space Administration.

The paper is in final form and no version of it will be published elsewhere. 
this can presently be done to astrophysically interesting thresholds for both ground and space-based instruments.

The frequency band in which a ground-based interferometer can be made most sensitive to gravitational waves [5] ranges from about ten Hertz to about a few kilohertz, with arm lengths ranging from a few tens of meters to a few kilometers. Space-based interferometers, such as the coherent microwave tracking of interplanetary spacecraft [3] and proposed Michelson optical interferometers in planetary orbits [4] are most sensitive to millihertz gravitational waves, with arm lengths ranging from $10^{6}$ to $10^{8}$ kilometers.

In the Doppler tracking technique a distant interplanetary spacecraft is monitored from Earth through a microwave tracking link, and the Earth and the spacecraft act as free falling test particles. A radio signal of nominal frequency $\nu_{0}$ is transmitted to the spacecraft, and coherently transponded back to Earth where it is received and its frequency is compared to a radio signal of frequency referenced to a highly stable clock (typically a Hydrogen maser). Relative frequency changes $\Delta \nu / \nu_{0}$ as functions of time are then measured. A gravitational wave propagating through the solar system causes small perturbations in $\Delta \nu / \nu_{0}$ which are replicated three times in the Doppler data with maximum spacing given by the two-way light propagation time between the Earth and the spacecraft. This characteristic signature of the Doppler response, referred to as the three-pulse response, was first derived in its general form in 1975, by Estabrook and Wahlquist [6]. In the following Section we outline its derivation, and generalize it to a configuration with $B$ bounces.

In Section 3 we describe the different noise sources affecting the Doppler data, and provide a summary of the upper limits identified by the Doppler tracking technique to gravitational wave amplitudes in the millihertz band. In Section 4 we present our remarks and conclusions.

2. The response functions to a gravitational wave pulse. The net effect of a weak gravitational wave train on the frequency of a coherent radio signal transponded once in a stationary, freely falling, configuration of source and transponder is the so called three-pulse response function [6]. A gravitational wave pulse contributes to the interferometrically measured frequency change at three times, namely at the time it is incident on the source, the intermediate time when the radio signal bounces off the spacecraft, and at the round-trip light time.

In this Section we will deduce the general expression for the phase shift due to a gravitational wave when an electromagnetic beam is made to bounce $B$ times between two freely falling (geodesic) particles [7]. These could be the Earth and a spacecraft or, as in the more usual situation of an Earth-based interferometer, two highly reflective mirrors forming an optical cavity.

In this Section we will interchangeably refer to microwaves and transponders or laser light and mirrors, since the two configurations can be considered equivalent as far as the derivation of the response function to a gravitational wave pulse is concerned. Let us therefore consider a source of electromagnetic radiation, a laser for instance, to be at the first mirror, and the net frequency change, or equivalent phase fluctuation, to be 
interferometrically measured there. Let us also consider the following space-time metric

$$
d s^{2}=-d t^{2}+(1+h) d x^{2}+(1-h) d y^{2}+d z^{2},
$$

where $h=h(t-z) \ll 1$. To first order, this is the general relativistic solution for the strain field of a linearly polarized gravitational wave train propagating in vacuum along the positive $z$ direction. The metric could be generalized by adding in an amplitude for the other possible polarization, but to first order it is just as easy to do this at the conclusion, as needed. Let us also assume that our two particles are stationary in the $(x, z)$ plane. We will denote by $\alpha$ the cosine of the angle between the direction of propagation of the gravitational wave and the line joining particle $a$ to particle $b$, and $L$ their relative distance.

In this space-time the two particles follow a geodesic motion, represented by world lines parallel to the $t$ axis. We can visualize our physical system in a space-time diagram in which the vertical axis is the time $t$, while the horizontal axis is the line $\alpha z+\beta x$ (with $\beta^{2}=1-\alpha^{2}$ ). The $t$ axis coincide with the world line $x=y=z=0$ of particle $a$, while the world line for particle $b$ is (to first order in $h$ ): $x=\beta L, y=0$, and $z=\alpha L$. The characteristic wave fronts of the gravitational wave are given by $t-z=$ constant.

Consider, at an arbitrary time $t$, a perfectly monochromatic photon of frequency $\nu_{0}$ (as measured in the rest frame of $a$ ) emitted at $a$, which bounces off the end particle $b$ at time $t+L$, and then returns to particle $a$ at time $t+2 L$. The trajectory of the photon in this space-time is represented by two null geodesics, one originating at the event that we label 0 (on the world line of $a$ ) and ending at the event labelled 1 on the world line of $b$; the other connects the event 1 to the event labelled 2 back to the world line of $a$. Parallel transport of a null vector along these null geodesic is used to calculate $\nu_{1}$, the frequency measured at event 1 in the rest frame of $b$, and $\nu_{2}$ at event 2 again in the rest frame of $a$.

The frequency shifts $\nu_{1}-\nu_{0}$, and $\nu_{2}-\nu_{1}$ are related to the gravitational wave amplitude according to the following simple "two-pulse" relationships $[6,7]$

$$
\begin{gathered}
\frac{\nu_{1}(t+L)}{\nu_{0}}=1+\frac{(1+\alpha)}{2}[h(t)-h(t+(1-\alpha) L)], \\
\frac{\nu_{2}(t+2 L)}{\nu_{1}(t+L)}=1+\frac{(1-\alpha)}{2}[h(t+(1-\alpha) L)-h(t+2 L)],
\end{gathered}
$$

where $\nu_{0}$ is independent of time, since for the moment we are considering a monochromatic light source (or "atomic" frequency standard)

If we multiply together Eq. (2) and Eq. (3), and disregard second order terms in the wave amplitude $h$, we deduce the three-pulse response function in its original form [6]

$$
\frac{\nu_{2}(t+2 L)}{\nu_{0}}=1+\frac{(1+\alpha)}{2} h(t)-\alpha h(t+(1-\alpha) L)-\frac{(1-\alpha)}{2} h(t+2 L),
$$

Eq. (4) is then best rewritten to display the fractional frequency change at $a$ as a function of time $t$

$$
y(t) \equiv \frac{\nu_{2}(t)-\nu_{0}}{\nu_{0}}=-\frac{(1-\alpha)}{2} h(t)-\alpha h(t-(1+\alpha) L)+\frac{(1+\alpha)}{2} h(t-2 L) .
$$


The phase difference $\Delta \phi^{(1)}(t)$ measured, say, by a photo detector, is related to the corresponding frequency change (Eq. (5)) as follows

$$
y(t)=\frac{1}{2 \pi \nu_{0}} \frac{d \Delta \phi^{(1)}(t)}{d t} .
$$

If we define the Fourier transform of the time series $\Delta \phi^{(1)}(t)$ to be given by

$$
\widetilde{\Delta \phi^{(1)}}(f) \equiv \int_{-\infty}^{+\infty} \Delta \phi^{(1)}(t) e^{2 \pi i f t} d t
$$

we can rewrite Eq. (5) in the Fourier domain as

$$
\frac{\widetilde{\Delta \phi^{(1)}}(f)}{2 \pi \nu_{0}}=-\frac{R(\alpha, f)}{2 \pi i f} \widetilde{h}(f)
$$

In Eq. (8) $R(\alpha, f)$ is the three-pulse transfer function

$$
R(\alpha, f)=-\frac{(1-\alpha)}{2}-\alpha e^{2 \pi i(1+\alpha) f L}+\frac{(1+\alpha)}{2} e^{4 \pi i f L} .
$$

For those who prefer to think in terms of heterodyne detection, of signals on a carrier of amplitude $A_{0}$ and frequency $\nu_{0}$, this phase modulation engenders side bands of amplitude $A$ given by

$$
\frac{A\left(\nu_{0}+f\right)}{A_{0}}=\frac{\nu_{0}}{f}\left[R(\alpha, f) R(\alpha, f)^{*}\right]^{1 / 2} \widetilde{h}(f) .
$$

If we expand Eq. (9) in the long wavelength limit $(f L \ll 1)$, to first order in $f L$ Eq. (8) becomes [6]

$$
\frac{\widetilde{\Delta \phi^{(1)}}(f)}{2 \pi \nu_{0}} \simeq\left(\alpha^{2}-1\right) L[1+\pi i(\alpha+2) f L] \widetilde{h}(f) .
$$

The factor $\left(\alpha^{2}-1\right)$ is the "beam pattern" of a single-bounce linear gravitational wave antenna. In the long wavelength limit, its "antenna gain" is $\approx L$.

Let us now assume that the light between the two particles makes $B$ bounces before it is made to interfere with the light of the laser. We want to determine what the corresponding phase change will be in this case. It is easy to see that the frequencies $\nu_{2}(t+2 L)$, $\nu_{3}(t+3 L)$, and $\nu_{4}(t+4 L)$, for instance, are related among themselves as $\nu_{0}, \nu_{1}(t+L)$, and $\nu_{2}(t+2 L)$ assuming proper care of the time argument is taken. We can for example find that the following expression for $\nu_{4}(t+4 L) / \nu_{2}(t+2 L)$ holds

$$
\frac{\nu_{4}(t+4 L)}{\nu_{2}(t+2 L)}=1+\frac{(1+\alpha)}{2} h(t+2 L)-\alpha h(t+2 L+(1-\alpha) L)-\frac{(1-\alpha)}{2} h(t+4 L) .
$$

If we multiply Eq. (4) by Eq. (12) we get, to first order in $h$,

$$
\begin{aligned}
\frac{\nu_{4}(t+4 L)}{\nu_{0}}=1 & +\frac{(1+\alpha)}{2} h(t+2 L)-\alpha h(t+2 L+(1-\alpha) L)-\frac{(1-\alpha)}{2} h(t+4 L) \\
& +\frac{(1+\alpha)}{2} h(t)-\alpha h(t+(1-\alpha) L)-\frac{(1-\alpha)}{2} h(t+2 L) .
\end{aligned}
$$


If we use the definition of $y(t)$ given in Eq. (5), Eq. (13) can be rewritten in the following way

$$
\frac{\nu_{4}(t)-\nu_{0}}{\nu_{0}}=y(t)+y(t-2 L) .
$$

After some simple algebra we can easily deduce the following expression for the frequency change after $B$ bounces

$$
\frac{\nu_{2 B}(t)-\nu_{0}}{\nu_{0}}=\sum_{k=0}^{B-1} y(t-2 k L) .
$$

Let us now denote by $\Delta \phi^{(B)}(t)$ the phase shift measured at the photo detector for the $B$ bounce configuration. Taking into account Eq. (15), we can write the following equation

$$
\frac{1}{2 \pi \nu_{0}} \frac{d \Delta \phi^{(B)}(t)}{d t}=\sum_{k=0}^{B-1} y(t-2 k L)
$$

which in the Fourier domain becomes

$$
\frac{\widetilde{\Delta \phi^{(B)}}(f)}{2 \pi \nu_{0}}=-\frac{\widetilde{y}(f)}{2 \pi i f} \sum_{k=0}^{B-1} e^{4 \pi i k f L} .
$$

From the definition of $y(t)$ (Eq. (5)), and after adding the geometric progression, we can rewrite Eq. (17) as

$$
\frac{\widetilde{\Delta \phi^{(B)}}(f)}{2 \pi \nu_{0}}=-\frac{R(\alpha, f) \widetilde{h}(f)}{2 \pi i f}\left[\frac{1-e^{4 \pi i B f L}}{1-e^{4 \pi i f L}}\right] .
$$

If we expand Eq. (18) in the long wavelength limit, that is to say when $f L \ll 1$ but allowing $B$ to be large enough that $4 B f L \simeq 1$, for the dominant frequency band of the gravitational wave signal, we get

$$
\frac{\widetilde{\Delta \phi^{(B)}}(f)}{2 \pi \nu_{0}} \simeq \frac{\left(1-\alpha^{2}\right)}{2} \frac{\left(1-e^{4 \pi i B f L}\right)}{2 \pi i f}[1+\pi i(\alpha+2) f L] \widetilde{h}(f)
$$

Note that the transfer function given in Eq. (19) does not increase linearly with the arm length, as it did for the one-bounce configuration, $B=1$. For a given arm length $L$ and for a gravitational wave signal of dominant frequency $f$, we can choose the number of reflections $B$ in such a way that $4 B f L \simeq 1$, and the response is optimal, depending only on $f$ and the geometrical factor $\left(1-\alpha^{2}\right)$.

Note that this condition also holds for a Michelson interferometer, since its transfer function is essentially equal to the one given in Eq. (19), apart from a different antenna pattern $[8,9]$. At one kilohertz an orthogonal-arm interferometer, of 40 meters arm length and $B \simeq 2000$ bounces, would experience the same phase shift due to a passing gravitational wave as would an interferometer of 4 kilometer arm length and $B \simeq 20$ bounces.

3. Noise sources and their transfer functions. Substantial effort over the past fifteen years has been devoted to the understanding of the noise sources affecting spacecraft Doppler tracking, and to estimate their strengths and spectral properties [10]. In what follows we will summarize the main noise sources by providing their power spectral 
densities, $S_{y}(f)$, as well as by expressing their amplitudes in terms of the Allan Deviation. For an arbitrary random process $y(t)$, the Allan Variance estimated at the integration time $\tau$ is defined by the following expression

$$
\sigma_{y}^{2}(\tau)=\frac{\left\langle[\bar{y}(t+\tau)-\bar{y}(t)]^{2}\right\rangle}{2}
$$

where $\bar{y}(t)$ is defined as follows

$$
\bar{y}(t)=\frac{1}{\tau} \int_{t}^{t+\tau} y(s) d s
$$

and angle brackets denote time averages.

Noise sources affecting the sensitivity of Doppler tracking experiments can be divided into two broad classes: (i) Instrumental and (ii) Propagation.

3.1. Instrumental noise sources. In the high frequency region of the band accessible to Doppler tracking, thermal noise dominates over all other noise sources at about $10^{-1}$ $\mathrm{Hz}$. This noise is white in phase, being determined essentially by the finite temperature of the receiver and the finite intensity of the signal. In frequency the power spectral density therefore grows with the square of the Fourier frequency, $S_{y}(f) \propto f^{2}$, making this noise source the dominant one in the "blue" region. Since this noise appears at the moment of detection $t$, its transfer function into the Doppler data is the identity.

Among all other instrumental noise sources (transmitter and receiver, mechanical stability of the antenna, stability of the spacecraft transponder, spacecraft buffetting, irregularities of the spacecraft spin rate, micro seismic disturbances, instabilities introduced by signal distribution within the ground station, clock noise, etc.) clock noise has been shown to be the most important instrumental source of frequency fluctuations [10].

Let us define $C(t)$ to be the random process associated with the relative frequency fluctuations introduced by the clock. As a consequence of the definition of the Doppler observable $\left(\frac{\Delta \nu(t)}{\nu_{0}}\right)$ measured on the ground, we conclude that $C(t)$ shows up at two different times, namely under the linear combination $C(t-2 L)-C(t)$. This time signature can be understood by observing that the frequency of the signal received at time $t$ contains clock fluctuations transmitted $2 L$ seconds earlier. By subtracting from the received frequency the frequency of the radio signal transmitted at time $t$, we also subtract clock frequency fluctuations with the net result shown above. Since the power spectral density [10] of $C(t)$ goes as $f^{-1}$, we deduce that the spectral density of the clock noise in the Doppler data has the following dependence on the Fourier frequency $f$ :

$$
S_{y}(f) \propto \frac{\sin ^{2}(2 \pi f L)}{f}
$$

The noise level of a typical atomic standard, like the Hydrogen Maser used by the Deep Space Network (DSN), has been measured to be about $7.0 \times 10^{-16}$ at 1000 seconds integration time.

3.2. Propagation noise sources. The radio link to the spacecraft crosses regions of space in which the index of refraction $n(t, \vec{r})$ is different from one, and changes in space 
and time. The propagation noise is due to fluctuations in the index of refraction of the troposphere, ionosphere, and the interplanetary solar plasma.

As the radio signal to the spacecraft crosses the Earth's troposphere, it suffers a path delay $\Delta L$. The time variations of $\Delta L$ induce frequency shifts $\Delta \nu$, of the main carrier frequency $\nu_{0}$ :

$$
\Delta L=\int_{\text {raypath }}[n(t, \vec{r})-1] d s ; \Delta \nu=\frac{\nu_{0}}{c} \frac{d}{d t} \Delta L(t),
$$

where the main contribution to the integral is limited to a region around the Earth.

It is important to say that at microwave frequencies the index of refraction of tropospheric irregularities does not depend on the carrier frequency [10]. In other words the noise level due to the troposphere is independent of the frequency $\nu_{0}$.

Let us define $T(t)$ to be the random process associated with the frequency noise due to the troposphere. From the definition of the Doppler observable we have that $T(t)$ enters into the Doppler data at two different times through the linear combination $T(t-2 L)+$ $T(t)$. This is because the frequency of the received signal is affected at the moment of reception as well as $2 L$ seconds earlier. Since the frequency of the signal generated at time $t$ does not contain yet any of these fluctuations, it follows that $T(t)$ is positive-correlated [10] at the round trip light time $2 L$. Since the power spectral density [10] of $T(t)$ goes approximately as $f^{-1 / 2}$, we deduce that the spectral density of the troposphere noise in the Doppler data has the following dependence on the Fourier frequency $f$ :

$$
S_{y}(f) \propto \frac{\cos ^{2}(2 \pi f L)}{\sqrt{f}}
$$

The contribution of the tropospheric noise to the overall Allan Deviation at 1000 seconds can be as large as $1.0 \times 10^{-13}$, depending on the local weather conditions.

Ionosphere and interplanetary plasma have been the dominant noise sources in Spacecraft Doppler tracking experiments analyzed so far, in which S-Band $(\approx 2 \mathrm{GHz}) \mathrm{mi}-$ crowave links were used. Since the plasma index of refraction at microwave frequencies scales as $\nu_{0}^{-2}$, these noise sources can be suppressed by increasing the microwave frequency. Furthermore, by collecting data at solar opposition, that is to say when the Sun-Earth-Probe angle is larger than about $160^{\circ}$, the plasma wind remains in the radio path much longer, minimizing in this way the variations in the index of refraction.

From plasma scintillation data it has been shown [11] that the spectral density of this noise has a power law dependence, $S_{y}(f) \propto f^{-\beta}$, where $\beta \epsilon[0.6,1.0]$. The contribution of the plasma noise to the overall noise budget has the following magnitude

$$
\sigma_{y}(1000) \approx 4 \times 10^{-14}\left(\frac{2.3 \mathrm{GHz}}{\nu_{0}}\right)^{2}
$$

From the considerations made above we can write the Doppler response $y(t)$ to a gravitational wave pulse as the following rather complete expression

$$
\begin{aligned}
y(t)= & -\frac{(1-\mu)}{2} h(t)-\mu h(t-(1+\mu) L)+\frac{(1+\mu)}{2} h(t-2 L)+ \\
& +C_{E}(t-2 L)-C_{E}(t)+2 B(t-L)+T(t-2 L)+T(t)+ \\
& +A(t-2 L)+T R(t-L)+E L(t)+P(t),
\end{aligned}
$$


where the random processes $B, A, T R_{s c}, E L$, and $P$ correspond respectively to the noise due to the antenna and buffeting of the spacecraft, the radio amplifier on the ground, the spacecraft transponder, the electronics on the ground, and the interplanetary plasma.

Data taken with the spacecraft Viking, Voyager, Pioneer 9, Pioneer 10, Pioneer 11, Mars Observer, Ulysses, Galileo have been, and will be, analyzed in search for bursts, continuous, and a stochastic background of gravitational radiation [11-15]. Although none of the searches performed so far has led to a detection, upper bounds for the strength of various possible gravitational waveforms have been obtained. In what follows I will summarize the most stringent limits set to date on bursts, sinusoids and chirps, and a stochastic background of gravitational radiation.

3.3. Bursts. Gravitational wave bursts could be generated at a detectable level in the Doppler tracking band by collapses to form black holes, or by collisions of two black holes [5]. Most of the searches performed so far have been done by using data from spacecraft tracked at S-Band. Since the signal-to-noise ratio, after applying matched filters, depends on the differences between the spectral characteristics of the signal searched for and the noise [10], an independent and objective measure of the upper limits set by these experiments on burst radiation is given by the Allan Deviation. A reference sensitivity to bursts was set by the Pioneer 11 spacecraft, which was tracked in 1983. The Allan deviation of the measured noise was about $7 \times 10^{-14}$, being determined entirely by plasma scintillation [10].

In March 1993 the spacecraft Mars Observer was tracked for about three weeks at $\mathrm{X}$-Band $(8.4 \mathrm{GHz})$. Due to the higher frequency of the radio link the plasma noise was reduced by almost a factor of 20 with respect to the S-Band experiments. From a preliminary analysis of the data, an Allan Deviation of $1.0 \times 10^{-14}$ has been measured, making Mars Observer the most sensitive Doppler tracking experiment to date [16].

3.4. Sinusoids and chirps. Binary systems containing super massive black holes are the candidate sources of sinusoids and chirps gravitational waves in the millihertz frequency band [5]. The best sensitivities to these signals have been set by the Pioneer 10 experiment in December 1993, and by the spacecraft Ulysses during the February 1992 experiment. The Ulysses spacecraft was tracked for three weeks at S-Band, and it also had an X-Band radio signal in the down-link. Because of the two frequencies in the down-link, a perfect calibration of the dispersive plasma noise in the down-link was possible. The Pioneer spacecraft was tracked instead for almost 30 days, but only at S-Band. The one-sigma limits set by these experiments were $7.0 \times 10^{-15}$ and $3.5 \times 10^{-15}$ respectively [17, 18].

An analysis of the data taken in 1993 with Mars Observer will be performed soon, in search for this class of signals. The expected sensitivity at one sigma level is expected to be about $10^{-15}$.

3.5. Stochastic background. The spectrum of the signal in the Doppler data results from averaging the three-pulse response function over all possible directions [6]. The analytic dependence of such a spectrum is almost like the noise spectrum of the reference clock, having a sinusoidal modulation. This modulation is fortuitous, since it is basically orthogonal to the modulation of the leading noise sources, namely ionospheric and tro- 
pospheric noise. This difference between the signal and the noise has been exploited to reach an almost clock-noise-limited upper limit for the gravitational wave background spectrum at selected frequencies [10]. The best upper limit have been deduced only recently with the 1993 Pioneer 10 experiment. This spacecraft was tracked in December 1993 for almost 30 days, at S-Band and with a round-trip-light-time of about 16 hours. It was therefore possible to search for a gravitational wave background at low frequencies, just below $10^{-5} \mathrm{~Hz}$. Since the local average of the spectrum, around the frequencies where the propagation noise is minimum, has been measured to be $1.2 \times 10^{-23} \mathrm{~Hz}^{-1}$, the corresponding one sigma sensitivity has been estimated to be a few parts in $10^{-14}$.

4. Conclusions. Spacecraft Doppler tracking is the only existing experimental technique that allows searches for gravitational waves in the millihertz frequency band. Although the sensitivities achieved so far have been relatively modest, it is important to note that all the experiments performed so far have utilized radio components that were not purposely designed for gravitational wave searches. For the first time, frequency stability requirements on the radio system on board the spacecraft and the ground antenna have been approved on the upcoming NASA project Cassini. This interplanetary spacecraft will be launched in October 1997 for gathering scientific data from Saturn, Titan and other natural satellites of the Saturnian system. During part of the spacecraft trajectory (cruise and opposition), from year 2001 until year 2003, for forty days each year, searches for gravitational radiation will be performed. The implementation of a purposely designed radio system with frequency stabilities specified by the experimenters, indicate that an Allan deviation equal to $7.0 \times 10^{-16}$ should be achievable.

\section{References}

[1] Y. Gürsel, P. Linsay, R. Spero, P. Saulson, S. Whitcomb, and R. Weiss. In: A study of a long Baseline Gravitational Wave Antenna System, National Science Foundation Report (1984).

[2] LIGO Project, California Institute of Technology, Massachusetts Institute of Technology (U.S.A.). In: Proposal to the National Science Foundation, A Laser Interferometer Gravitational-Wave Observatory (LIGO), Volumes 1 and 2, 1989 (unpublished).

[3] H. D. Wahlquist, J. D. Anderson, F. B. Estabrook, and K. S. Thorne. In: Atti dei Convegni dei Lincei, 34, 335 (1977).

[4] LISA: Laser Interferometer Space Antenna for the detection and observation of gravitational waves. A Cornerstone Project in ESA's long term space science program Horizon 2000 Plus, Pre-Phase A Report, MPQ 208, (December 1995).

[5] K. S. Thorne, in 300 Years of Gravitation, edited by S. W. Hawking and W. Israel (Cambridge University Press, Cambridge, England, 1987).

[6] F. B. Estabrook and H. D. Wahlquist, Gen. Relativ. Gravit. 6, 439 (1975).

[7] M. Tinto and F. B. Estabrook, Phys. Rev. D, 52, 1749 (1995).

[8] J.-Y. Vinet, B. Meers, C. N. Man, and A. Brillet, Phys. Rev. 38, 433 (1988).

[9] K. S. Thorne (unpublished notes).

[10] J. W. Armstrong. In Gravitational Wave Data Analysis, ed. B.F. Schutz, Kluwer, Dordrecht, (1989). 
[11] J. W. Armstrong, R. Woo, and F. B. Estabrook, Ap.J.,230, 570 (1979).

[12] R. W. Hellings, Phys. Rev. D, 23, 844, (1981).

[13] J. D. Anderson, J. W. Armstrong, F. B. Estabrook, R. W. Hellings, E. K. Lau, and H. D. Wahlquist, Nature, 308, 158, (1984).

[14] J. D. Anderson, and B. Mashoon, Ap.J., 290, 445, (1985).

[15] J. W. Armstrong, F. B. Estabrook, and H. D. Wahlquist, Ap.J., 318, 536, (1987).

[16] J. W. Armstrong: private communication, (1995).

[17] J. D. Anderson, J. W. Armstrong, and E. Lau, Ap.J., 408, 287, (1993).

[18] B. Bertotti, R. Ambrosini, J. W. Armstrong, et al. Ap.J., 296, 13, (1995). 